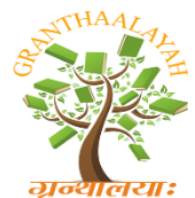

INTERNATIONAL JOURNAL OF RESEARCH GRANTHAALAYAH A knowledge Repository

Science

\title{
CORRELATION BETWEEN SIZE OF LEFT LOBE OF THE LIVER AND BODY CHARACTERISTIC AMONG SUDANESE PATIENTS 2018-2019
}

\author{
Maha Esmeal Ahmed Esmeal ${ }^{1}$, Nagla Hussien Mohamed Khalid ${ }^{2}$ \\ ${ }^{1} \mathrm{Ph}$. D, Associate Professor, Radiography and Medical Imaging Sciences, Radiological Sciences \\ College, National University, Sudan \\ ${ }^{2}$ Ph. D, Assistant Professor, Department Radiological Sciences, Applied Medical College, \\ Najran University, Kingdom of Saudi Arabia
}

\begin{abstract}
Background: The liver is that the largest organ within the anatomy. the dimensions depends on many factors: age, sex, body size and shape, from all the specific examination technique utilized Ultrasonography of the liver is one of the most common routine investigations which assess the size, texture and pathological change.

Objective: The aim of this study was to Study the correlation between size of left lobe of the liver and body characteristic in Sudanese patients sono-graphically.

Method: The data used in this study was collected in a randomly selected population sample and identify factors that affect liver size in Khartoum hospitals, the data collected from august 2018 to august 2019.

Result: 100 subjects (42 male-58 female) underwent sonographic examination of the liver in the mid clavicular line to determine liver span. The mean age of the sample was 36.8 (SD14.4), ranging from 20 to 87 years. The result of the study revealed that the mean of the liver span 15.02+_0.35, the mean of left lobe was 5.21+_0.39 and the mean of caudate lobe was $2.5+0.07$. The study also found that there is strong positive relation between body mass index and left lobe of the liver, but there was no such correlation between body mass index and liver span. Researchers recommended that a future study, continuous research in this subject is highly recommended.
\end{abstract}

Keywords: Liver Size; Body Characteristics; Left Lobe.

Cite This Article: Maha Esmeal Ahmed Esmeal, and Nagla Hussien Mohamed Khalid. (2019). "CORRELATION BETWEEN SIZE OF LEFT LOBE OF THE LIVER AND BODY CHARACTERISTIC AMONG SUDANESE PATIENTS 2018-2019.” International Journal of Research - Granthaalayah, 7(11), 19-27. 10.29121/granthaalayah.v7.i11.2020.330.

\section{Introduction}

The liver is a large solid gland that occupies the whole of the right hypochondrium greater part of the epigastrium and extends into the left hypochondrium reaching up to the left lateral line. The liver weighs about $1400 \mathrm{gm}$ to $1600 \mathrm{gm}$ in males and about $1200 \mathrm{gm}$ to $1400 \mathrm{gm}$ in females ${ }^{(1)}$. The 
liver and its companion biliary tree and gallbladder are considered together because of their anatomic proximity, their interrelated functions and the overlapping features of some of the diseases that affect these organs. Residing at the crossroads between the digestive tract and the rest of the body, the liver has the enormous task of maintaining the body's metabolic homeostasis. The structural unit of the liver is the liver lobule, a roughly hexagonal column of liver cells (hepatocytes) between adjacent lobules are branches of the hepatic artery and portal vein. The capillaries of a lobule are sinusoids, large and very permeable vessels between the rows of liver cells. The sinusoids receive blood from both the hepatic artery and portal vein, and it is with this mixture of blood that the liver cells carry out their functions. The hepatic artery brings oxygenated blood, and the portal vein brings blood from the digestive organs and spleen. Each lobule has a central vein. The central veins of all the lobules unite to form the hepatic veins, which take blood out of the liver to the inferior vena cava. The cells of the liver have many functions, but their only digestive function is the production of bile.

Bile enters the small bile ducts, called bile canaliculi, on the liver cells, which unite to form larger ducts and finally merge to form the hepatic duct, which takes bile out of the liver. The hepatic duct unites with the cystic duct of the gallbladder to form the common bile duct, which takes bile to the duodenum. Bile is mostly water and has an excretory function in that it carries bilirubin and excess cholesterol to the intestines for elimination in feces. The digestive function of bile is accomplish by bile salts, which emulsify fats in the small intestine. Emulsification means that large fat globules are broken into smaller globules. This is mechanical, not chemical digestion the fat is still fat but now has more surface area to facilitate chemical digestion. Production of bile is stimulated by the hormone secretin, which is produced by the duodenum when food enters the small intestine Ultrasonography of the liver is one of the most common routine investigations which assess the size, texture and pathological changes. It is the first line of investigation for differential diagnosis of suspected liver pathologies. The measurement of the liver with ultrasound is important and useful to assess the size. Alterations in the size of the liver occur in a wide variety of clinical conditions. It increases in some diseases like viral hepatitis, alcoholic liver disease, congestive cardiac failure, certain storage, and metabolic disorders, etc. and may diminish in some conditions as in acute fulminant hepatitis, cirrhosis, etc. or remain unchanged in number of diseases, assessment of liver size is a very important diagnostic clue for some diseases ${ }^{(2)}$. Routinely the liver enlargement is expressed by centimeters or fingers palpable below the right costal margin. The palpability of the liver below the thoracic cage still constitutes the common method for assessing its size. Liver size is best estimated clinically by liver span. The vertical distance between uppermost and lowermost points of hepatic dullness estimated by percussion in the right midclavicular line is the liver $\operatorname{span}^{(3)}$. The liver was routinely measured in the midclavicular line from the dome of the diaphragm and normally has a craniocaudally length of up to $16 \mathrm{~cm}$. Alength over $16 \mathrm{~cm}$ usually indicates significant hepatomegaly though the bulk of the patient and configuration of the liver should be considered. If the liver measures $16 \mathrm{~cm}$ or more it is abnormal in 75 percent of patients. Longitudinal measurement alone is 87 percent accurate in measuring hepatic size. As the liver enlarges it usually loses its sharp angular configuration becoming lobular in contour with a rounded inferior edge. If the liver length is, $13 \mathrm{~cm}$ or less it is considered to be normal in size in 93 percent of cases. Niederau et al studied the normal measurement of the liver with an ultrasound he reported that there was a weak correlation between liver dimensions against (longitudinal and transverse measurement) and physical data of the study population. He 
mentioned that longitudinal diameter of the liver alone will give too high or too low a value, respectively) ${ }^{(3,4)}$

Scanning of the viscera is carried out to know the normal dimensions and the echo patterns and deviations from normal have led to diagnosis or prediction of pathological conditions (Marco P, et al 2002). Liver size varies widely according to age. Many diseases can affect its size ranging from infective processes to malignant disorders. Palpation and percussion are the standard bedside techniques to document liver size but are far from accurate to detect a small increase in size ${ }^{(5)}$ The liver is the largest organ in the body and also the largest gland. It has been estimated that between birth and adulthood there is at least a 10 -fold increase in liver mass ${ }^{(6)}$. The size of the liver has been reported to be affected by intrinsic liver disease and other systemic pathologies $\left({ }^{7)}\right.$. Several studies have been conducted on the establishment of normal liver sizes in adults. ${ }^{(8)}$

Hepatomegaly is a frequent clinical finding in adult and can be caused by intrinsic liver diseases or systemic disorders ${ }^{(9)}$ which include malaria, inflammatory conditions like hepatitis, sickle cell anemia, tumors which can be primary or metastatic, infection from bacteria and parasites, poisons/toxins including ingestion of herbs and roots, acetaminophen, insecticide, and mushroom. Others include chronic cardiac failure, storage disorders, infiltrative disorders like leukemia, lymphoma, granulomas like tuberculosis autoimmune, non-alcoholic fatty liver, malnutrition like kwashiorkor and trauma (10). Hepatomegaly is a condition that needs urgent further evaluation (11) and ultrasound is usually the method of choice for screening diagnostic, prognostic purposes, and in the follow-up after treatment because of its accuracy and easy accessibility, avoids the use of ionizing radiation and the technique is non-invasive and cheap. The importance of early detection of an adult with liver disease cannot be overemphasized because it is critical for optimal medical or surgical intervention. A study done in Nigerian adults showed that liver disease accounts for $7.9 \%$ of medical admissions and liver cirrhosis and primary liver cancer account for two-thirds of the liver diseases in hospitalized patients in a Nigerian hospital. ${ }^{(1)}$

Primary liver cancer or hepatocellular carcinoma is the major cause of death by cancer worldwide, accounting for over half a million deaths per year (12). It is the second leading cause of cancerrelated deaths in the world (13). Hence, there is an urgent need for the establishment of sets of normative values for our environment .

Various methods for assessment of liver sizes have been reported in the literature ${ }^{(14)}$ some of which include a clinical evaluation by percussion, radiography and radionuclide studies, ultrasound, and computed tomography. The effectiveness of some of these methods has also been criticized ${ }^{(15)}$. Diagnostic imagining techniques have been reported to be much more superior to clinical examination in determining the size of the liver ${ }^{(2)}$. Clinical evaluation by percussion and palpation is flawed for its inability to provide precise liver dimensions. This procedure can only give a gross estimation of sizes with very high tendencies for overestimation due to the pleural effusion or lung consolidation or underestimation due to tympany in the right upper abdomen (2). Technetium liver scan has been criticized for its underestimation of the liver dimensions and radiography and radionuclide studies for exposure of patients to ionizing radiation (16). Computed tomography and Magnetic Resonance Imaging (MRI) have been noted to be expensive and uncommon in developing countries. ${ }^{(17)}$ 
Ultrasound and Computed tomography have been made popular by its accuracy, affordability, and portability. With the appearance of liver transplantation, it has become certain that there is an extraordinary requirement for a careful assurance of liver size. Determined volume estimations made at ultrasound or at computed tomography give, the best appraises. With computed tomography, 8 to 12 sequential transverse slice are acquired; a PC ascertains the encased region and the all-out organ volume is assessed. This technique gives determined liver volumes inside $5 \%$ of the real volume as decided at autopsy. An ongoing report utilizing sagittal section of the liver got by ultrasound was seen as better than transverse areas acquired witch computerized tomography on account of the absence of respiratory artifact when sagittal section are gotten (17). Further refinements of these and other technique may build exactness. In most of typical assessments, the liver is not obvious. Cases in which the ordinary liver is unmistakable incorporate emphysema, right-sided pleural effusion, slender body carriage, Riedel's lobe, or profound diaphragmatic outing (17)

In emphysema, the lungs are hyperactive extended with diaphragmatic smoothing, in this way bringing down the upper and lower borders of the liver. A huge right pleural effusion will have a similar impact of lowering the liver margins. Exact percussion of the upper liver outlying in a patient with a privilege pleural effusion can be troublesome due to the liquid thickness overlying liver bluntness. People with a profound diaphragmatic outing, for example, artists and continuance competitors may have palpable liver on inspiration ${ }^{(14)}$. The most widely recognized pathologies related with a palpable and enlarged liver incorporate metastatic malignancy, lymphoma, congestive cardiovascular breakdown, alcoholic hepatitis, and different reasons for fatty liver (jejunoileal bypass and complete parenteral nutrition). Cirrhosis might be related with a liver of small, ordinary, or increased size. In metastatic or primary liver tumor, the liver becomes infiltrated with deposit of malignant growth cells that can develop quickly. Such neoplastic development can generally be distinguished from the normal liver by palpation of hard and nodular tissue constancy (14). Comparable liver enlargement can happen in lymphoma. In cirrhosis the liver might be enlarged or small, the last for the most part happening in end-stage cirrhosis or fulminant hepatic failure following hepatocyte necrosis and collapse ${ }^{(2)}$ The consistency is normally nodular in cirrhosis. Traditionally it is micro nodular because of alcoholic or wholesome infection and macro nodular when cirrhosis is post hepatitis. Alcoholic hepatitis and different reasons for fatty liver lead to increase size of liver by hepatocyte fatty infiltration and hepatocyte enlargement hepatic distention and smooth enlargement are typical of important right-sided heart failure, which happens due to hepatic venous blockage auxiliary to impeded myocardial capacity ${ }^{(12)}$

Unlike most other organs the liver gets blood from 2 sources (dual blood supply) the hepatic artery supplies the liver with blood rich in oxygen from the heart and the portal vein brings nutrient-rich blood from the intestines .(13)

The liver is made up mainly of cells called hepatocytes. It also contains other types of cells including cells that line its blood vessels and cells that line small tubes in the liver called bile ducts. The bile ducts extend out of the liver and carry bile from the liver to the gallbladder or directly to the intestines. These different types of cells in the liver can form several types of malignant (cancerous) and benign (non-cancerous) tumors. These tumors have different causes are treated differently and have a different prognosis (13). Benign tumors sometimes grow large enough to cause problems, but they do not grow into nearby tissues or spread to distant parts of the body. If 
they need to be treated, the patient can usually be cured with surgery (13). The most common type of benign liver tumor hemangiomas starts in blood vessels. Most hemangiomas of the liver cause no symptoms and do not need treatment, but some may bleed and need to be removed surgically. Hepatic adenoma is a benign tumor that starts from hepatocytes (the main type of liver cell). Most cause no symptoms and do not need treatment. But some eventually cause symptoms such as pain or a mass in the abdomen (stomach area) or blood loss because there is a risk that the tumor could rupture (leading to severe blood loss) and a small risk that it could eventually develop into liver cancer most experts usually advise surgery to remove the tumor if possible. Using certain drugs may increase the risk of getting these tumors. Women have a higher chance of having one of these tumors if they take birth control pills although this is rare. Men who use anabolic steroids may also develop these tumors. Adenomas may shrink when the drugs are stopped ${ }^{(14)}$

Focal nodular hyperplasia $(\mathrm{FNH})$ is a tumor-like growth made up of several cell types (hepatocytes, bile duct cells, and connective tissue cells). Although FNH tumors are, benign it can be hard to tell them apart from true liver cancers and doctors sometimes remove them when the diagnosis is unclear. If you have symptoms from an FNH tumor, it can be removed with surgery. Both hepatic adenomas and FNH tumors are more common in women than in men. Cancer that starts in the liver is called primary liver cancer. There is more than one kind of primary liver cancer. Hepatocellular carcinoma is the most common form of liver cancer in adults. It is also sometimes called hepatoma. About 4 of 5 cancers that start in the liver are this type (1). Intrahepatic cholangiocarcinoma (bile duct cancer) about $10 \%$ to $20 \%$ of cancers that start in the liver are intrahepatic cholangiocarcinomas. These cancers start in the cells that line the small bile ducts (tubes that carry bile to the gallbladder) within the liver. Most cholangio carcinomas actually start in the bile ducts outside the liver. Although the rest of this document deals mainly with hepatocellular cancers cholangiocarcinomas are often treated the same way (12). Angiosarcoma and hemangiosarcoma are rare cancers that begin in cells lining the blood vessels of the liver. People who have been exposed to vinyl chloride or to thorium dioxide (Thorotrast) are more likely to develop these cancers. Some other cases are thought to be caused by exposure to arsenic or radium or to an inherited condition known as hereditary hemochromatosis. In about half of all cases, no likely cause can be identified. These tumors grow quickly and are usually too widespread to be removed surgically by the time they are found. Chemotherapy and radiation therapy may help slow the disease but these cancers are usually very hard to treat. These cancers are treated like other sarcomas ${ }^{(14)}$. Hepatoblastoma this is a very rare kind of cancer that develops in children. Usually in those younger than 4 years old. The cells of hepatoblastoma are similar to fetal liver cells. About 2 out of 3 children with these tumors are treated successfully with surgery and chemotherapy although the tumors are harder to treat if they have spread outside the liver ${ }^{(18)}$. Secondary liver cancer Most of the time when cancer is found in the liver it did not start there but has spread (metastasized) from somewhere else in the body such as the pancreas, colon, stomach, breast, or lung. Because this cancer has spread from its original (primary) site, it is secondary liver cancer. These tumors are named and treated based on their primary site (where they started). For example, cancer that started in the lung and spread to the liver is called lung cancer with spreads to the liver, not liver cancer and it is treated as lung cancer. In the United States and Europe, secondary (metastatic) liver tumors are more common than primary liver cancer. The opposite is true for many areas of Asia and Africa ${ }^{(18)}$. 


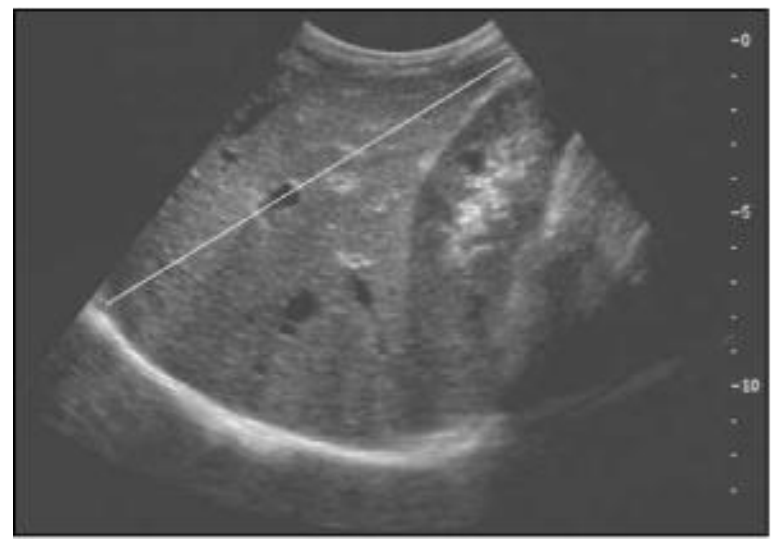

A

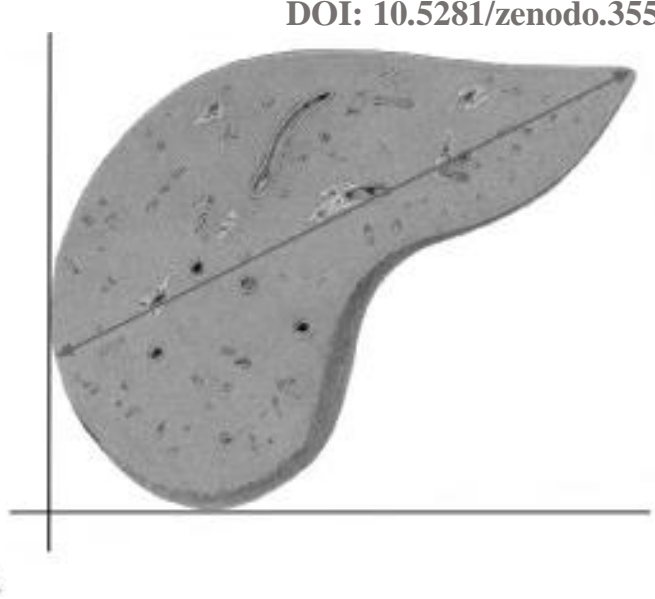

DOI: 10.5281/zenodo.3556854

Figure 1: liver span measurement

\section{Material and Methods}

Material \& Method: This a descriptive quantitate cross sectional study conducted in Sudan Khartoum state hospital. The tools of the study including a special sheet (closed questionnaire) Were being designed to collect the data. Samples analyzed in this study are consisted of 100 patients measured by ultrasonography radiologic tools.

Sample size: The sample size were selected using convenience-sampling technique The data used in this study was collected in a randomly selected population sample and identify factors that affect liver size in Khartoum hospitals, the data collected from august 2018 to august 2019.

Inclusion Criteria: male and female (20-87) years old. Exclusion criteria: male and female less than 20 years old and more than 87 years old. Variables of Data collection: Height, weight, BMI, age and Gender (demographic characteristic), body mass index, Size of left lobe, size of quadrate lobe and portal vein.

Methods of data analysis: The data analyzed using SPSS (statistical package of social science version 23).

Ethical approval: Ethical approval has been granted from the hospital and the department of radiology this did not include or disclose any [ID] information concerning the patient. Informed consent was obtained from all individual participants; included in the study.

\section{Results}

The following tables and figures present data that obtained from (100) patients who were examined by ultrasound 
Table 1: The mean and standard deviation to the body characteristics

\begin{tabular}{|l|c|c|c|c|c|c|c|}
\hline & $\begin{array}{c}\text { Age } \\
\text { (years) }\end{array}$ & Height(m) & BMI & $\begin{array}{c}\text { Liver } \\
\text { span }(\mathbf{c m})\end{array}$ & $\begin{array}{c}\text { Lt } \\
\text { Lobe } \\
\text { (cm) }\end{array}$ & $\begin{array}{c}\text { Caudate } \\
\text { Lobe } \\
(\mathbf{c m})\end{array}$ & $\begin{array}{c}\text { Portal Vein } \\
\text { Diameter (cm) }\end{array}$ \\
\hline Mean & 36.87 & 1.63 & 29.07 & 15.02 & 5.20 & 2.5 & 1.3 \\
\hline SD & 14.4 & 0.13 & 10.15 & 0.35 & 0.39 & 0.07 & 0.05 \\
\hline Min & 20 & 1.33 & 6.4 & 14 & 4.5 & 2.3 & 1.2 \\
\hline Max & 87 & 1.98 & 50.14 & 16 & 6.3 & 2.6 & 1.4 \\
\hline
\end{tabular}

Table 2: Correlation between the liver span, left lobe, and caudate lobe, portal vein with age, body mass index and height of the normal sample

\begin{tabular}{|l|c|c|c|c|}
\hline Variable & Liver span $(\mathbf{c m})$ & Lt Lobe $(\mathbf{c m})$ & Rt Lobe (cm) & Portal Vein Diameter (cm) \\
\hline Age & 0.034 & 0.511 & 0.111 & 0.586 \\
\hline BMI & 0.425 & 0.002 & 0.121 & 0.911 \\
\hline Height & 0.41 & 0.46 & 0.52 & 0.32 \\
\hline
\end{tabular}

\section{Discussion}

The general objective of this study was to establish a standard reference for liver size in healthy Sudanese by using ultrasound. The data of this study were collected from 100 healthy people (male-female) who underwent an abdominal ultrasound and the liver span left lobe, caudate lobe and portal vein were measured.

The results of this study reveal that the mean of the liver span $15.02+_{-} 0.35$, this is in agreement with, previous studies that found the mean ultra-sonic liver span to be $14.6 \mathrm{CM}$, although they reported a difference of almost two centimeters between males and females, it was not significant. (Kratzer Wolfgang et.al (2003) ${ }^{(19)}$ didn't stray far from the pattern when they found the mean liver span to be 14.0 CM. ( Monika Patzak et.al 2014) ${ }^{(20)}$ also came to the same overall measurement of 15.1 CM, and found a statistically significant correlation between liver span and gender, age, body mass index and body height, which this study failed to replicate in respect to height, this could be explained by sampling errors in our study, as their sample included 1,789 subjects versus 100 in the current study. This is supported by (Moawia Gameraddin et.al 2015) ${ }^{(21)}$, who also found Height to have a significant correlation with the liver span followed by weight in the normal subjects. Moawia Gameraddin et.al (2015) disagreed again with our study in the mean measurement of the left lobe, 9.4 CM, which is fairly high, compared to the mean in this study 5.21+_0.39, since the sample size is the same in both studies;it's probably attributed to the difference in actual sizes of the left lobe between Sudanese and Saudi population. The mean of the caudate lobe was 2.5+_0.07 and the mean of portal vein diameter was 1.3+_0.05 (table 4.1) this close enough to be called an agreement, again with (Monika Patzal et al 2014). This study also found that there is a strong positive relationship between body mass index and left lobe of the liver, but there was no such correlation between body mass index and liver span figure .furthermore, age did not show any substantial effects on the left lobe or caudate lobe. Neither did height on the liver span. (Niederau C, et al 1983) ${ }^{(22)}$ Concurred this when they reported a weak correlation between liver dimensions (longitudinal and transverse measurement) and physical data of the study population 


\section{Conclusion}

This study was an analytical cross-sectional design done to know the normal measurement of the liver size and to identify the relationship between these measurements and body characteristics in a sample of 100 healthy subjects. The study concluded that the mean of the liver span was $15.02+_{-}$ 0.35 , and that physical characteristic is a bad predictor of liver measurements. This study faced the limitation of the few participants and did not include all of the liver measurements, due to finical and logistical difficulties. Future studies with larger and more representative samples should be carried to establish normal baseline measurements for the Sudanese population. Researchers recommend that a 3-5 MHz curve linear probe must be used for liver scan and Portal vein measurement should always be included in every liver scan report for more accurate US exams .

\section{References}

[1] Chaurasia, B. D. Human anatomy regional and applied: Lower Limb and Abdomen. CBS Publishers, 1984.

[2] Rosenfield, Arthur T., Igor Laufer, and Peter B. Schneider. "The significance of a palpable liver: a correlation of clinical and radioisotope studies." American Journal of Roentgenology 122.2 (1974): 313-317.

[3] Abbas, K. \& Mitchell, F. (2007). Robbins Basic Pathology. $8^{\text {th }}$ ed. Elsevier.

[4] American Cancer Society. Cancer Facts \&Figures. Atlanta G.a: American Cancer Society; 2014

[5] American joint committee on cancer. liver. In: AJCC Cancer Stagging Manual . $7^{\text {th }}$ Ed. New York, NY, Springer; 2010:191-195

[6] Balistreri WF. Manifestation of liver disease. In: Behrmann RE, Kliegman RM, Jenson HB, Bartlett DL, DiBisceglie AM, Daswon LA, Cancer of the liver. In De vita VT, Lawrence TS, Rosenberg SA, (eds). De Vita, Hellman, AND Rosenberg Cancer Principles and Practice on Oncology. $9^{\text {th }}$ Ed. Philadelphia, Pa Lippincott Williams' \& Wilkins; 2011:997-1018.

[7] Bisset RA., Khan AN. Differential Diagnosis in Abdominal Ultrasound. London: W.B Saunders; 2000; pp.34.

[8] Borner N, Schwerk WB, Braun B. leber In: Braun B, Gunther R, Schwerk WB, (eds) UltraSchalldiagnostiK Landsberg, Germany: Ecomed; 1987:1-18

[9] C. Scanlon, V, \& Sanders, T. (2007). Essential of Anatomy and Physiology. $5^{\text {th }}$ ed. Philadelphia: F. A. Davis Company.

[10] Castell Do, Frank BB, Abdominal examination: role of percussion and auscultation. Postgrad Med 1977; 62(6):133.

[11] D. Boyer, T., L. Wright, T., \& P. Manns, M. (2006). Hepatology. $5^{\text {th }}$ ed., vol.1. Canada: Elsevier.

[12] Dhingra B, Sharma S, Mishra D, Kumari R, Pandy RM, Aggarwal S, normal value of Liver and spleen size by ultrasonography in Indian children. Indian pediatric 2010; 47:487-92

[13] Dittrich D, Milde S, Dinkel E, Baumann W, Weitezel D, Sonographic biometry of liver and spleen size in childhood. Pediatric Radiology 1983; 13 :206-11.

[14] Henderson JM, Heymesfield SB, Horowitz J, Kunter MH, measurement of liver and spleen volume by computed tomography. Assessment of reproducibility and changes found following a selective distal splenrenal shunt Radiology; 1981; 141:427

[15] Rumack, C.M. (2011). Diagnostic ultrasound. St. Louis: Elsevier Mosby.

[16] Sapira JD, Williamson DL. How big is the normal liver? Arch Intern Med1979; 139:971-973.

[17] Won chung, K. \& M. chung, H. (2012). Gross anatomy. $7^{\text {th }}$ ed. 201-203. Philadelphia: Lippincott Williams \& wilkins.

[18] Kratzer W1, Fritz V, Mason RA, Haenle MM, Kaechele (2003) Factors affecting liver size: a sonographic survey of 2080 subjects. 2003 Nov; 22(11):1155-61. Ultrasound Med.20 
[19] Monika Patzak,Marc Porzner,Suemeyra Oeztuerk,Richard Andrew MasonA SAkinli (2014):Assessment of Liver Size by Ultrasonography: Journal of Clinical Ultrasound 42(7).

[20] Moawia Gameraddin, Amir Ali, Mosleh Al-radaddi, Mohaned Haleeb, Sultan Alshoabi. The Sonographic Dimensions of the Liver at Normal Subjects Compared to Patients with Malaria. International Journal of Medical Imaging. Vol. 3, No. 6, 2015, pp. 130-136. doi: 10.11648/j.ijmi.20150306.14.

[21] Claus Niederau, Claus Niederau, J E Müller, W P Fritsch, T Scholten and W P Fritsch (1983): Sonographic measurements of the normal liver, spleen, pancreas, and portal vein: Radiology 149 (2): $537-40$.

*Corresponding author.

E-mail address: Najlabashab@ yahoo.com 